\title{
Depth-dependent Dynamics of Liquid Metal Surfaces with First Principles Simulations.
}

\author{
Beatriz G. del Rio, Luis E. González \\ Departamento de Física Teórica, Universidad de Valladolid, Paseo Belén 7, E-47011 Valladolid, SPAIN
}

\begin{abstract}
Liquid metal surfaces have gained increased interest over the last decade due to new applications in synthesis of 2D materials, catalysis, or fusion reactors. Static properties such as the reflectivity and density profile have been determined, both experimentally and computationally, for numerous liquid metals and alloys. However, the characterization of the dynamic properties has remained a challenging task and only one experimental study by Reichert et al. [Phys. Rev. Lett. 98 (2007) 096104] has evaluated the depth-dependence of different dynamic properties in the liquid indium (l-In) surface. In this paper, we present an ab inito molecular dynamics study of the collective dynamic properties of this same system at different depths, obtaining very good agreement with the experimental data. In addition, we are able to compute the properties much closer to the surface than experimentally attainable, and have discovered that at these shallower depths, the properties drastically differ from those deeper in the slab. Therefore, this study sheds light into the behavior of dynamic properties at the atomic interface and highlights the ability of ab initio molecular dynamics to study such unknown dynamic behavior of liquid metals surfaces at depths not yet attainable experimentally but of crucial importance for liquid surface physics.
\end{abstract}

Keywords: liquid surface, sound velocity, longitudinal viscosity, liquid In, ab initio simulations

\section{Introduction}

Applications such as synthesis of new materials [1, 2], energy storage [3-5], microfluidics [6, 7], flexible electronics $[8,9]$, and drug delivery [10] among many others [11], have propelled liquid metals to a position of high interest over the last years. However, although the study of the dynamic properties of bulk liquid metals has advanced considerably, both experimentally and theoreticaly, it is not the case for liqud metal surfaces. The main objective of experimental studies of liquid metal surfaces over the last 30 years has been to determine static structural properties such as the X-ray reflectivity curve, and the density profile associated to it. In 1981, D'Evelyn and Rice [12] predicted that the atomic density near the free surface of a metallic liquid would change in a non monotonic way, displaying a stratification in layers parallel to the interface. Those oscillations would show up in the X-ray (XR) reflectivity curve, $R\left(q_{z}\right)$, as a Bragg-like peak located at a wave vector $q_{z} \approx 2 \pi / \lambda$, where $\lambda$ is the spacing between layers. However, it wasn't until 1995 when Magnussen and coworkers [13] reported the existence of surface layering in liquid $\mathrm{Hg}$ $(\mathrm{l}-\mathrm{Hg})$ at room temperature. Since then, XR reflectivity measurements have been performed on other liquid metal surfaces, such as Ga, In, K, Sn and Bi [14-18], showing in

Email address: luisen@metodos.fam.cie.uva.es (Luis E. González) all cases the expected Bragg-like peak in the reflectivity. From a theoretical standpoint, both classical and ab initio studies, have also focused on these static properties, confirming the available experimental data, and also sampling systems harder to handle experimentally.

Liquid metals present a great number of other interesting surface phenomena, whose influence on the dynamic properties in the near-surface region is largely unknown, especially at short length scales. The collective dynamics of a bulk liquid metal encompass the existence of longitudinal and transverse waves, where acoustic and shear modes, respectively, propagate and disperse, like phonons in the solid phase. However, in a slab configuration other types of waves may appear, for instance, capillary waves $(\mathrm{CW})$ [19] in which particles perform a circular movement as a combination of oscillations both longitudinal and transverse to the surface. Experimental techniques like inelastic neutron scattering or inelastic X-ray scattering (IXS) allow the study of the dynamics in bulk liquids at interatomic length scales. These studies have provided important insights concerning the nature of propagating excitations (sound modes), their relation to the local atomic structure, and the influence of relaxation phenomena on the excitation spectrum [20]. However, the high-frequency dynamics of liquid surfaces and interfaces is still a largely unexplored field. In fact, only one experimental work by Reichert and coworkers [21, 22], has investigated the microscopic dynamics at the liquid surface of In, by using IXS 
in grazing angle geometry. By varying the incident angle around the critical angle of total reflection, they were able to selectively study the bulk and surface dynamics from a probing depth of $4 \mathrm{~nm}$ to $180 \mathrm{~nm}$. They reported the influence of $\mathrm{CW}$ in the spectra as an additional contribution with a Lorentzian shape, especially for small momentum transfers and at the smallest probing depth of $4 \mathrm{~nm}$. They also reported on an increase of the longitudinal viscosity by a factor of two in the near surface region, but no significant change in the adiabatic sound velocity.

Computationally, the study on the dynamic properties of liquid metal surfaces is also extremely scarce. The first (and only) molecular dynamics (MD) study of the dynamical properties of a liquid surface was performed by Iarlori et al. [23] on a slab of liquid gold using classical MD. They predicted a sound velocity $20-30 \%$ smaller than in the bulk liquid and observed the existence of CW in both the longitudinal and transverse collective dynamics. From the point of view of $a b$ initio (AI) MD simulations, the study of the collective dynamics of free liquid surfaces has never been performed, given the high computational demand of such calculations because of the large number of particles required. This is due to two reasons: first, good statistics for the dynamic properties of the layers requires a large number of atoms in each layer, and second, while a depth of $4 \mathrm{~nm}$ is a short length for experiments, it is huge for standard AIMD simulations.

Prompted by the experiments on the l-In surface, we have performed the first AIMD study on the dynamical properties of a free liquid surface. By using orbital-free (OF)-AIMD, we have been able to simulate a large enough sample (3000 particles) for tens of picoseconds, making it possible to study the collective dynamics of the system at various depths from the surface, including single-layer regions not attainable experimentally.

\section{Methodology}

Given a set of ions and valence electrons, the total energy of the system can be calculated, within the BornOppenheimer approximation, as the sum of the classical kinetic energy of the ions, the Coulomb ion-ion repulsion and the electronic ground state energy. This last term can be computed accurately through density functional theory (DFT) [24, 25], which states that it is the minimum of a functional of the electron density, $n(\vec{r})$, given as

$$
E[n(\vec{r})]=T_{s}[n(\vec{r})]+E_{\text {ext }}[n(\vec{r})]+E_{H}[n(\vec{r})]+E_{x c}[n(\vec{r})]
$$

where $T_{s}$ represents the kinetic energy of non-interacting electrons, $E_{\text {ext }}$ is the interaction of the electrons with the potential created by the ions, $E_{H}$ is the Hartree term due to the electrostatic repulsion between the electrons, and $E_{x c}$ is the electron exchange-correlation (XC) energy. We have specifically considered the OF-DFT version, where $T_{s}[n(\vec{r})]$ is given as a kinetic energy density functional
(KEDF) which depends explicitly on the density. Despite being less accurate than the orbital-based KohnSham scheme, this method allows the computational study of much larger samples for much longer times. In particular we have used a kinetic energy functional put forward by Gonzalez et al [26, 27], which has convenient mathematical, computational and physical properties. It was derived from the averaged density approach of Chacon, Alvarellos and Tarazona [28] and Garcia-Gonzalez et al. [29-31], and is given as

$$
\begin{gathered}
T_{s}=T_{v W}[n]+T_{\beta} \\
T_{\beta}[n]=\frac{3}{10} \int d \vec{r} n(\vec{r})^{5 / 3-2 \beta} \tilde{k}(\vec{r})^{2} \\
\tilde{k}(\vec{r})=\left(3 \bar{k}_{F}\right)^{3} \int d \vec{s} k(\vec{s}) w_{\beta}\left(2 \bar{k}_{F}|\vec{r}-\vec{s}|\right) \\
k(\vec{r})=\left(3 \pi^{2}\right)^{1 / 3} n(\vec{r})^{\beta}
\end{gathered}
$$

with $\bar{k}_{F}$ the Fermi wavevector corresponding to a reference mean electron density $\bar{n}$, and $w_{\beta}(x)$ representing a rigid weight function, determined by requiring the correct recovery of the linear response limit at the reference mean density and the Thomas-Fermi limit. More details can be found in Refs. [26, 27].

In the present AIMD simulation we use the local density approximation [32] for $E_{x c}$, and the $E_{\text {ext }}$ term is represented by using pseudopotentials. The orbital-free character of the theory requires the ion-electron interaction to be described by a local pseudopotential (LPS), dependent only on the electron-ion distance. Local pseudopotentials, in contrast with non-local ones used in orbital-based schemes, can face limitations to correctly represent localized features in the valence electron density, caused by covalent bonding or $d$ electrons. In order to alleviate these limitations, we have followed here a two-phase procedure for constructing an accurate LPS to correctly represent the liquid slab of In. In a first step we constructed an initial LPS using the neutral pseudoatom (NPA) method [33, 34], where the environment of an atom in the liquid is modeled as a jellium. This jellium representation of the enviroment is more realistic than vacuum, but in a real liquid ions are not homogenously distributed around a central one. A correction to include information on the liquid environment is introduced in a second step involving the use of a force-matching (FM) process [35], using as benchmark the forces obtained for several configurations taken from a "small" Kohn-Sham (KS-) AIMD simulation of a l-In slab, whose details are given in Appendix A, that comprises 205 atoms that interact with the valence electrons via a nonlocal pseudopotential (NLPS). The matching of the forces is achieved by modifying the LPS with the addition of gaussians, using a simulated annealing optimization [36]. The parameters varied in the optimization process are the number of gaussians and their positions, amplitudes and widths, with the restriction that the $q=0$ derivative of the peudopotential is zero. 


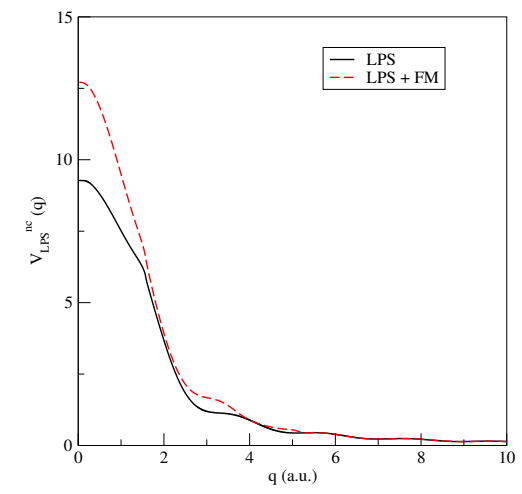

Figure 1: Non-coulombic part of the LPS before (black) and after (dashed red) force-matching.
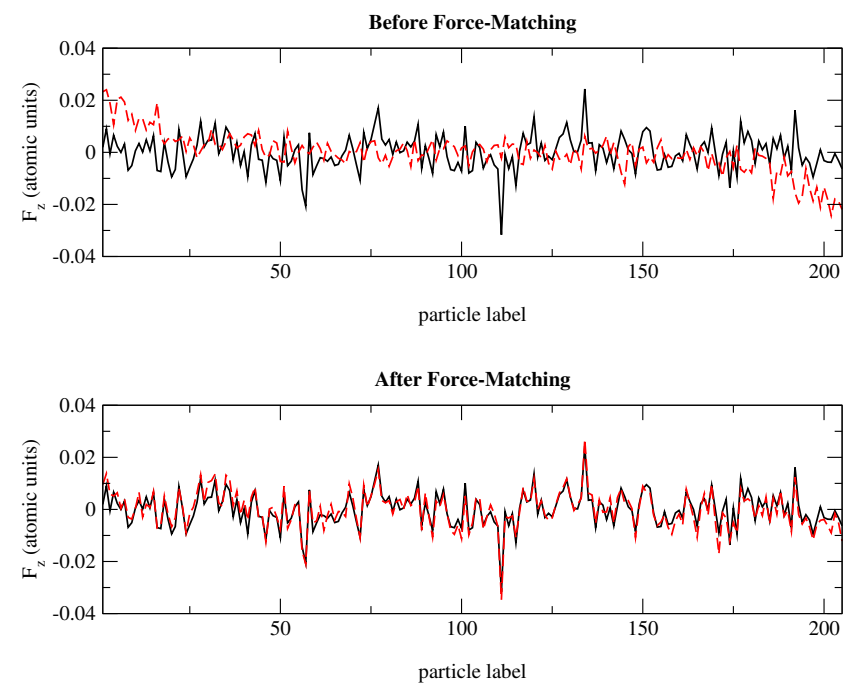

Figure 2: $z$-components of the atomic forces calculated with KSDFT/NLPS (black) and with OF-DFT/LPS (dashed red), before (top) and after (bottom) force-matching. The particles are labeled by increasing $z$ coordinate.

Figure 1 shows the initial LPS computed with the NPA method and the final LPS obtained after FM. As can be seen in the figure, the final pseudopotential is larger everywhere than the NPA original one. In particular the initial value at $q=0$ suffers a significant increase in order to better represent the interactions in the liquid slab, but nevertheless this is just a consequence of the FM procedure and not imposed externally to the optimization.

Figure 2 displays the $z$-component of the forces on the atoms for a configuration not used in the fitting, obtained by the KS-DFT calculation with a NLPS and by the OFDFT calculation using the LPS obtained before (top) and after (bottom) the FM process. The difference in the $z$ components of forces between KS-DFT/NLPS and OFDFT/LPS before FM is evident, especially at both interfaces. However, after FM the forces representation is drastically improved. The same occurs for the $x$ and $y$ components of the forces, which are not shown, but the

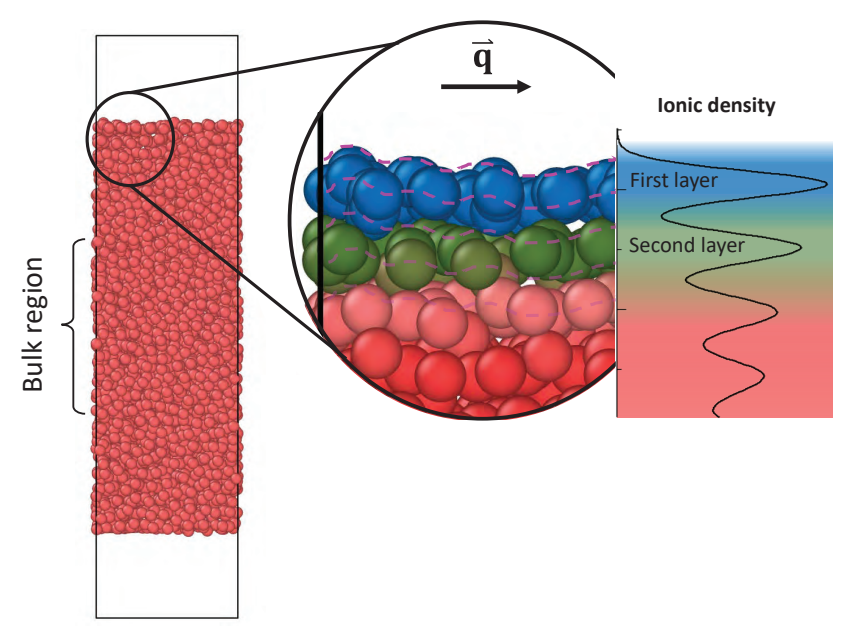

Figure 3: Sketch of the simulation setup. In the zoomed area $\vec{q}$ is a characteristic wavevector of the dynamic properties studied, and the colors indicate the belonging of the atoms to different layers, whose contributions to the dynamic properties change gradually as suggested in the rightmost graph (see section 4). The outermost wiggled line portraits pictorically the liquid-vacuum separation surface used to obtain the intrinsic profile and reflectivity (see section 3).

improvement is not so significant, since the differences between these components in the two calculations are much smaller than those of the $z$ component shown in figure 2 . This is due to the fact that the interface in the $z$ direction is ultimately responsible for the relaxation of the slab, that affects largely the $z$ components of the forces.

Note that ionic motion is determined by the forces, and therefore reproducing the correct forces guarantees a correct representation of the system both structurally and dynamically.

With the selected KEDF and the LPS thus constructed we have performed OF-AIMD simulations of the free liquid surface of In at $550 \mathrm{~K}$, somewhat above the melting point of $429 \mathrm{~K}$ [37], with an initial density of $\rho=0.0369$ $\AA^{-3}[38]$. The system contained 3000 atoms in a supercell of dimensions $L_{x}=L_{y}=29.254 \AA$ and $L_{z}=120 \AA$, with two free surfaces normal to the $z$ axis. A sketch of the simulation setup is given in figure 3 . The initial width of the slab was of $95 \AA$, which guarantees about $25 \AA$ separation between periodic images of the slab, and can accomodate a sampling depth that allows for a direct comparison with the experimental data $[21,22]$ at the lowest probing depth experimentally attainable in the study, $40 \AA$. We generated 20000 equilibrium configurations with a time step of $4 \mathrm{fs}$, so the total equilibrium simulation lasts for $80 \mathrm{ps}$.

\section{Static structure}

Prior to the determination of the dynamic properties of the system we have compared the static structure obtained in our simulations with the experimentally measured one. The huge difference between sample sizes in experiments and simulations requires the comparison to be performed 
very carefully, since the effects of thermally excited CW at the surface are completely different in magnitude. The proper comparison is made at the level of the so called intrinsic surface structure factor, $\left|\Phi_{\text {int }}\left(q_{z}\right)\right|^{2}$, which portraits how the structure would be in the absence of CW.

The experimental determination of $\left|\Phi_{\text {int }}\left(q_{z}\right)\right|^{2}$ proceeds through X-ray reflectivity measurements, followed by transformations based on the macroscopic capillary wave theory [19], which take into account the surface tension, the temperature, the geometry of the experimental setup and an intrinsic surface roughness (details in Appendix B).

In atomistic simulations the determination of the intrinsic surface structure factor proceeds through its definition in terms of the Fourier Transform of the derivative of the intrinsic electronic density profile [19]. Since the simulation sample is obviously non-macroscopic we have chosen to follow an intrinsic sampling method (ISM) [39] in order to eliminate the CW microscopically through the determination of a reference separation surface, at each simulation step, that represents the rigged surface produced by the CW. The distances from the atoms to this surface are then used to construct the intrinsic density profile (IDP) that is free of CW effects at the atomic scale (see details in Appendix C). This method was already applied successfully to analyze the surface structure of liquid K [40]

Figure 4 depicts the average density profile (ADP), obtained directly from the atomic $z$ coordinates, and the IDP evaluated following the ISM procedure. The stratification of the density profile in layers close to the liquid surface is clearly observed. The interlayer distance is $\lambda=2.68 \AA$, in very good agreement with the experimental data of 2.69 $\AA$ [15]. The inset of figure 4 shows the X-ray reflectivity evaluated from the electronic IDP obtained from a superposition of the atomic electron densities centered at each ionic position, using the $\mathrm{CW}$-free separation surface. The results show an excellent agreement with experiment [15], presenting a characteristic peak located around $q_{z} \simeq 2.3$ $\AA^{-1}$, which coincides with the corresponding peak due to the interlayer distance in the IDP, $2 \pi / \lambda \simeq 2.34 \AA^{-1}$. This very good comparison confirms our correct static representation of the l-In surface.

\section{Collective dynamics}

To study the collective dynamic properties at different depths of the l-In slab, only the particles located at the region of interest must be used in the calculation of the properties. However, during their evolution, particles can move inside or outside the region at any time, and this can lead to a discontinuous time variation of the properties. To avoid this undesired behavior, we have instead multiplied the contribution of each particle to a dynamic magnitude by a weight function, $W_{\text {reg }}(z)$ (see Appendix D), whose value is one inside the region of interest and decays smoothly to zero within a small specified distance outside that region, which has been taken as the interlayer distance, i.e. $2.68 \AA$. Thus, for instance, the number

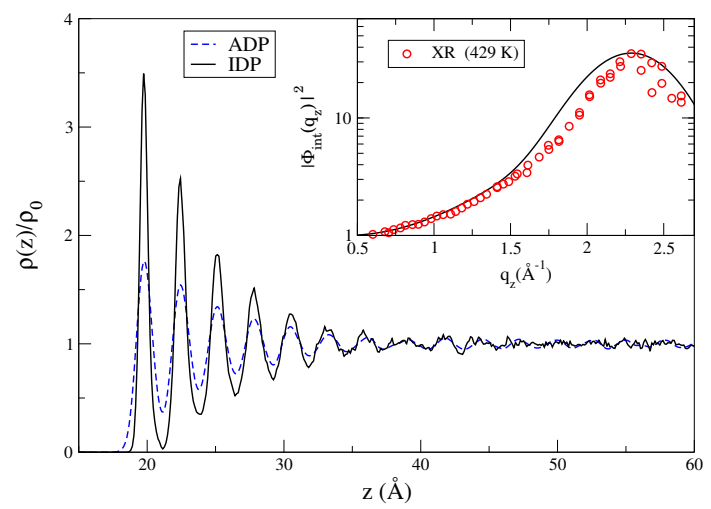

Figure 4: OF-AIMD ADP (dashed blue) and IDP (black). Inset: Intrinsic surface structure factor from OF-AIMD (black) and experimental XR reflectivity data (circles) [15].

of particles in the chosen region is $N_{\text {reg }}=\sum_{i=1}^{N} W_{\text {reg }}\left(Z_{i}\right)$, the corresponding particle density in reciprocal space is $\rho_{\text {reg }}(\vec{q})=\sum_{i=1}^{N} W_{\text {reg }}\left(Z_{i}\right) \exp \left[-i \vec{q} \cdot \vec{R}_{i}\right]$, and so on.

Below we report results obtained at three different regions, namely, the fisrt layer of the interface, the second layer, and finally a wide section in the interior of the slab that we call the bulk region.

In addition, in order to make a proper comparison with the IXS experimental data, we have also used an exponential weight function, $W_{\exp }(z)$, that mimics the exponential decay of the x-ray intensity inside the sample. We have chosen the decay rate so as to have a probing depth of 40 $\AA$, as in the experimental study.

The collective dynamics in each region are studied through the intermediate scattering function,

$$
F_{\text {reg }}(q, t)=\left\langle\frac{\rho_{\text {reg }}(\vec{q}, t) \rho_{\text {reg }}(-\vec{q}, 0)}{\sqrt{N_{\text {reg }}(t) N_{\text {reg }}(0)}}\right\rangle
$$

Consistently with the experiment, among all the wavevectors allowed by the periodic boundary conditions we consider only those parallel to the surface, so the average above refers to time origins and to those wavevectors that have the same module and $q_{z}=0$.

The Fourier Transform of $F(q, t)$ is the dynamic structure factor, $S(q, \omega)$, which can be compared with the experimental IXS intensity after performing several transformations: first it is converted into a quantum dynamic structure factor, $S_{Q}(q, \omega)$ that takes into account the quantum mechanical nature of the interaction between x-rays and the sample (detailed balance condition), then it is convoluted with the experimental energy resolution, and finally it is multiplied by an ad-hoc factor to take into account the arbitrary units in which the experimental results are reported (see Appendix E).

Figure 5 proves the agreement between our OF-AIMD results and the experimental data, that confirms our good representation of the liquid dynamics. There is an obvious mismatch in the height of the central peak between the OF-AIMD data and the experimental results for the 


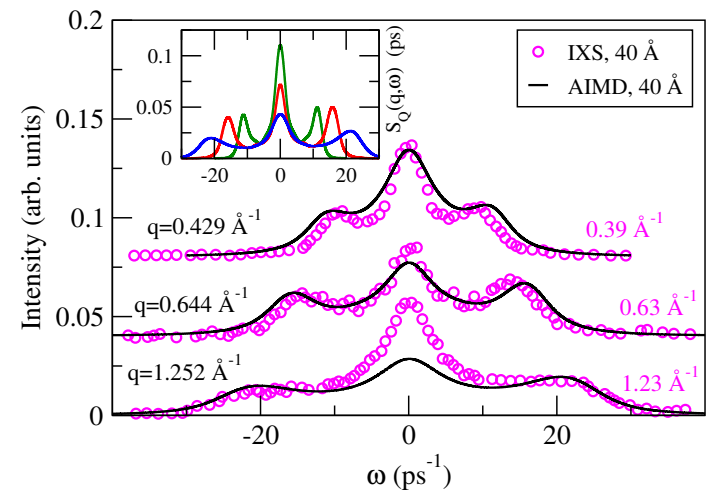

Figure 5: IXS data (circles) and OF-AIMD results (lines) for a $4 \mathrm{~nm}$ depth probe, for different wavevectors: right pink values from IXS and left black values for OF-AIMD results. The results for decreasing wavevectors are displaced upwards for clarity. The inset shows the OF-AIMD quantum dynamic structure factor, $S_{Q}(q, \omega)$, without convolution and in absolute units (ps). The green, red and blue curves correspond to the values of $q$ of the main graph in increasing order.

largest $q$ shown. We cannot give a definite reason for this discrepancy, but in the inset of the figure we observe that the evolution of $S_{Q}(q, \omega)$ as $q$ increases is quite smooth in the OF-AIMD results. This suggests that the experimental data may contain some spurious contribution in this central peak. In any case the characteristic features of the inelastic side peaks are very well reproduced. We will later analyze these characteristics in order to extract from them the depth-dependent thermophysical properties of interest.

Based on the good reproduction of both the experimental static and the dynamic structure, we can analyze confidently the region-dependent results as obtained by using the different weight functions. The results are shown in figure 6 both in its raw form and after convolved with the experimental resolution function. The OF-AIMD data reveal clear sound waves, whose amplitude is quite small at the first layer especially for the smaller $q$-values. Moreover we find clear indications of propagating waves of lower energy, that are nothing but the CW. As could be expected, these CW contributions are more evident at the first layer and for the smaller $q$-values.

It is enlightning to compare the OF-AIMD results obtained from the exponential weight function (black curves in figure 6), which as seen in figure 5 are similar to the experimental ones, with those corresponding to the bulk region (red curves in figure 6). They are almost indistinguishable at $q=1.252 \AA$ and very similar at the lower $q=0.644 \AA$. It is only for the lowest $q$ shown in the figure that the two curves are clearly different. This means that as soon as the wavevector increases above say $0.6 \AA$ the evanescent wave captures the properties of the bulk region, and only for smaller $q$ the experimental signal has a significant contribution from the surface region (and yet from the bulk region too). The OF-AIMD simulations,

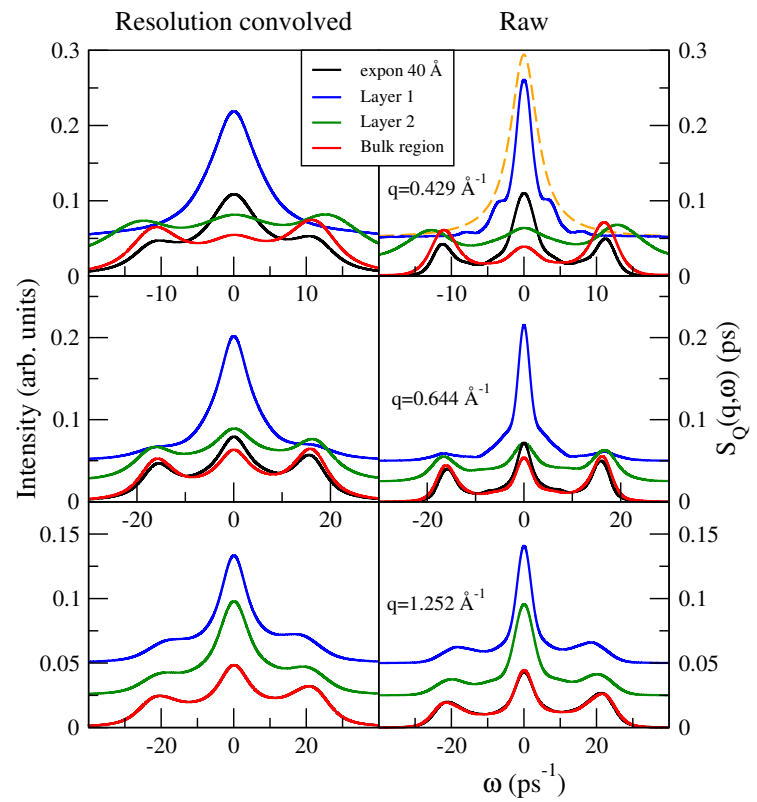

Figure 6: OF-AIMD quantum-corrected dynamics structure factors at several wavevectors before (right) and after (left) convolution with the experimental resolution function, which is shown (scaled) by the dashed line. Results for the first and second layer are displaced upwards.

on the contrary, can capture the specific region of interest no matter where it stands, and the results for the second layer, and even more for the outermost one, display strong differences with the bulk data for all the $q$ values shown.

The effects of the convolution are also observed to be very large, washing out all the low-energy features observed in the dynamic structure factor, especially at the level of the first layer, including relatively large values of $q$. This is in fact consistent with the experimental treatment of the CW as quasielastic Lorentzians.

In the hydrodynamic region $(q \rightarrow 0)$ the position and width of the side peaks of $S(q, \omega)$ are dictated respectively by the adiabatic sound velocity, $c_{s}$, and the attenuation factor, which includes terms from thermal diffusivity, $D_{T}$, and longitudinal viscosity, $\eta_{L}$, weighted through the specific heats ratio, $\gamma=C_{p} / C_{v}$. In $F(q, t)$ these thermophysical parameters determine the frequency and the decay rate of the oscillating term associated to sound propagation. Thermal relaxation amounts here to a simple exponentially decaying function whose rate is related to $D_{T}$. Going to larger wavevectors, within the generalized hydrodynamic theory, amounts to assign a $q$ dependence to these magnitudes. For a proper description of the dynamics of the slab we have already observed that the addition of another oscillating term is required due to the propagating capillary waves.

In order to study the variation of these thermophysical parameters, in particular $c_{s}(q)$ and $\eta_{L}(q)$, with depth, we have fitted the OF-AIMD $F(q, t)$ to a function including an exponentially decaying function plus two damped os- 


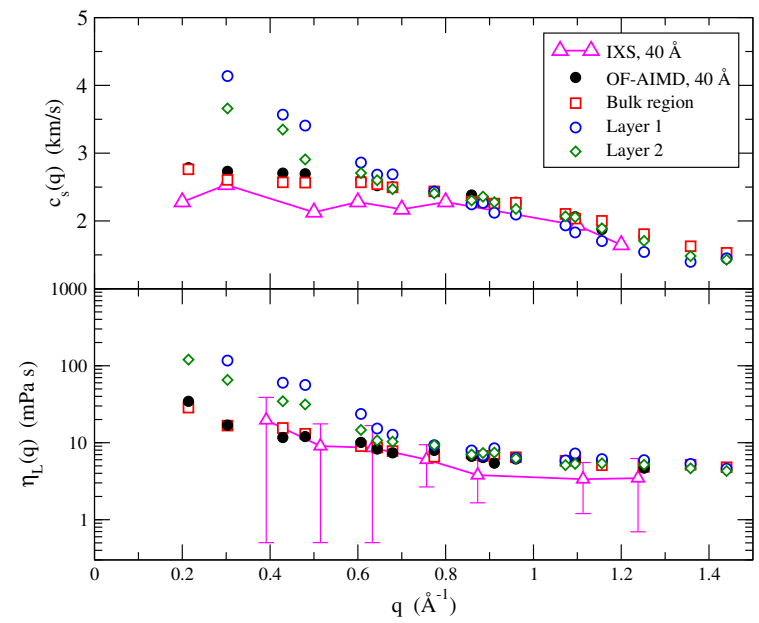

Figure 7: Comparison between IXS $[21,22]$ and OF-AIMD data for the $q$-dependent adiabatic sound velocity (top) and longitudinal viscosity (bottom). Note that IXS values for $\eta_{L}$ at $q<0.3 \AA$ could not be resolved with enough accuracy.

cillating functions, one for each type of wave (sound and $\mathrm{CW}$ ). In the experimental study this type of fits was also performed although using a different model (see details in Appendix F). In particular, a non propagating expression was used for the $\mathrm{CW}$ term as we have already mentioned above. In figure 7 we compare both calculations that correspond to $40 \AA$ probing depth, and in general a good agreement is observed, except for some overestimation of the adiabatic sound velocity. It must also be noted that the exponential probe and the results from the bulk region are almost identical.

However, clear differences appear in the behavior of these magnitudes at the first and second layers that can be captured only in the OF-AIMD simulation: as $q$ decreases, the values at both outer layers rapidly increase, reaching higher values than in the bulk region, by $50 \%$ in $c_{s}(q)$, and up to an order of magnitude in $\eta_{L}(q)$ (note the logarithmic scale) at $q=0.3 \AA^{-1}$. These results highlight the importance of AIMD studies to access information on various properties that are not yet experimentally accessible, but of critical importance in surface physics.

In order to understand the possible reasons behind these increases in $c_{s}$ and $\eta_{L}$ we have calculated additional properties of the layers, such as their average density and their transversal pair distribution function, $g_{T}(r)$, shown in figure 8. As a baseline comparison, we have included the results for a layer in the bulk region with the same width as the others, and denoted as central layer. The $g_{T}(r)$ of the central layer is basically identical to the bulk result, as detailed in the Supplementary Material (SM). We observe that as we move towards the interface the layers become gradually more structured, since the peaks and minima in $g_{T}(r)$ become more prominent. Moreover we observe an abrupt increase in the average density at the outermost layer. Both magnitudes therefore suggest a more "solid-
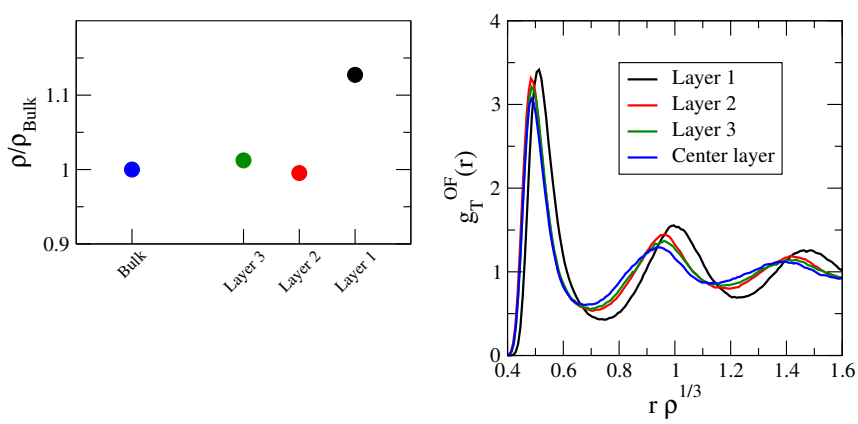

Figure 8: Average densities and transverse structure of the different layers upon approaching the interface. The distances are scaled by the corresponding density of each layer in order to take into account its variation.
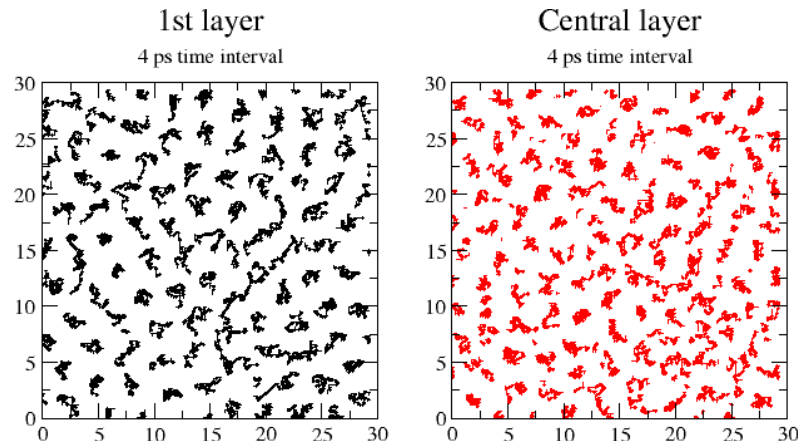

Figure 9: Trajectories of the particles of the first and the central layer during 4 ps of time.

like" behavior when approaching the surface, which is consistent with the observed increase in the thermophysical magnitudes reported above.

Nevertheless it should be understood that the layers do not solidify and the atoms still diffuse, as we portrait in figure 9 by comparing the trajectories of the atoms of the outermost layer with those of the atoms of the central layer. The same type of diffusive motion is clearly observed in both layers.

\section{Conclusion}

We have shown that accurate OF-AIMD simulations of large (from an ab initio perspective) liquid slabs are feasible, producing results in very good agreement with experimentally available data. Moreover, this work clearly demonstrates the value of such accurate OF-AIMD simulations in the investigation of collective dynamic properties at regions very close to the free liquid surface, still unreachable for IXS experiments, where large variations of thermophysical transport properties can take place. For the case of liquid In studied here these variations amount to a $50 \%$ increase in the sound velocity and an order of magnitude increase in the longitudinal viscosity. These results underlie the interest of performing this type of study 
for other liquid metallic surfaces. The experimental work on the dynamics of the l-In surface opened a very interesting field of research. However, much effort is still needed to reduce the probing depth to a few $\AA$ in order to measure the effect of the interface on the properties with respect to the bulk. In addition, higher resolution would be needed to observe experimentally the dispersive nature of capillary waves.

\section{Declaration of competing interest}

The authors declare that they have no known competing financial interests or personal relationships that could have appeared to influence the work reported in this paper.

\section{Acknowledgments}

We acknowlegde the funding by Junta de Castilla y Leon (Project VA124G18). LEG additionally thanks the support of the Spanish Ministry of Economy and Competitiveness (Project PGC2018-093745-B-I00), partially funded by FEDER.

\section{Appendix A. Benchmark simulation of an In slab}

The development of the LPS used in the final calculations includes a phase where a force-matching process [35] is performed. This ensures a correct representation by the LPS of the interaction between the different ions in the bulk liquid state as well as in the slab configuration. We used as benchmark for the fitting a KS-DFT simulation of l-In consisting of 205 atoms at $450 \mathrm{~K}$, performed with the Quantum Espresso package [41] and a non-local pseudopotential (NLPS). The details of the pseudopotential used are given in the SM. To perform the simulation of the slab we started with a bulk simulation where the 205 atoms were enclosed in an orthothombic box of sides $L_{x}=L_{y}=14.7 \AA, L_{z}=25.73 \AA$, such that the density matches the experimental one. This bulk simulation is also useful to test for the adequacy of the NLPS used, by comparison of the bulk results obtained with the experimental counterparts. Such checks are detailed in the SM. Subsequently to the bulk simulation we increased the length of the box in the $z$-direction to $33.72 \AA$, while keeping the atomic positions, thus forming the two interfaces, and let the system evolve for $4.23 \mathrm{ps}$, using a timestep of 7.5 fs. Finally, five configurations were randomly selected to be used as input in the FM process to minimize the difference between the atomic forces for those configurations calculated with KS-DFT using the NLPS and with OF-DFT using the LPS.
Appendix B. Experimental reflectivity and instrinsic surface structure factor.

The measured reflectivity upon sending x-rays of wavelentgh $\zeta$ onto a liquid surface at an angle $\alpha$ can be written as a product of three terms:

$$
R_{m}\left(q_{z}\right)=R_{F}\left(q_{z}\right)\left|\Phi_{\text {int }}\left(q_{z}\right)\right|^{2} \exp \left(-\sigma_{c}^{2} q_{z}^{2}\right),
$$

where $q_{z}=(4 \pi / \zeta) \sin \alpha$ is the momentum transfer perpendicular to the interface. $R_{F}\left(q_{z}\right)$ is the Fresnel reflectivity of a perfectly flat surface, and the quantity $\left|\Phi_{\text {int }}\left(q_{z}\right)\right|^{2}$ is the intrinsic surface structure factor, directly related to the Fourier Transform of the intrinsic total electron density profile, $n_{e, \text { int }}^{T}(z)$, which includes both valence and core electrons

$$
\Phi_{\mathrm{int}}\left(q_{z}\right)=\frac{1}{n_{e 0}^{T}} \int_{-\infty}^{\infty}\left(\frac{\partial n_{e, \mathrm{int}}^{T}(z)}{\partial z} \exp \left(-i q_{z} z\right)\right) d z
$$

where $n_{e 0}^{T}$ is the mean bulk total electron density. The term "intrinsic" refers to the resulting quantity when the effect of thermally excited capillary waves (CWs) are removed. These CWs appear as height fluctuations of the surface and produce a smearing of the density profiles and, correspondingly a depletion of the reflectivity, which is accounted for by the last term in (B.1). In that equation $\sigma_{c}$ is the surface roughness, usually written in terms of two contributions, $\sigma_{c}^{2}=\sigma_{0}^{2}+\sigma_{\mathrm{CW}}^{2}$, where $\sigma_{0}$ is an instrinsic surface roughness and $\sigma_{\mathrm{CW}}$ is quantified within the macroscopic CW theory [19],

$$
\sigma_{\mathrm{CW}}^{2}=\frac{k_{B} T}{2 \pi \gamma} \ln \left(\frac{q_{\max }}{q_{\min }}\right)
$$

with $\gamma$ the surface tension, and $q_{\max }$ and $q_{\text {min }}$ representing the largest and smallest wavevector contributions to the thermal excitation of the interface, respectively. A customary choice is $q_{\max }=\pi / a$ with $a$ being the ionic radius, whereas $q_{\text {min }}$ depends in general on the details of the experimental setup.

\section{Appendix C. Intrinsic sampling from computer simulations}

In atomic simulations, the sample size is extremely smaller than in experiments and consequently, the abundance of CWs is much reduced, but not negligible. Therefore, a proper comparison with experimental data needs to account for this difference. However, the application of the same macroscopic theory of CWs previously described to simulation samples of microscopic size can present serious shortcomings; a more direct access to the intrinsic profile and reflectivity is desirable. One such method was proposed by Chacon and Tarazona [39]. This procedure, named intrinsic sampling method (ISM) relies on 
the computation, for each MD configuration step, of the liquid-vapor separation surface, $z=\xi(x, y)$. The histogram of the particles distances to this separation surface along the $z$-direction is called the intrinsic density profile, with the CWs now removed at the atomic scale. The new CW-free $n_{e, \text { int }}^{T}$ allows for the calculation of the instrinsic surface structure factor from atomic simulations through Eq. (B.2), which can be compared with the experimental intrinsic surface structure, obtained through Eq. (B.1).

The procedure to determine the separation surface has several phases. Initially a set of a few (typically 9) pivot atoms is selected from a partitioned cell (in our case a $3 \times 3$ partition) by their $z$-coordinate. Next a surface $\xi_{0}(x, y)$ is constructed that passes through the pivot atoms and has a minimum change (with respect to $L_{x} L_{y}$ ) in the area as calculated to second order in the height. Then new pivot atoms are included and new surfaces $\xi_{i}(x, y)$ are constructed with the same criterion as before. The new pivot in each phase is the atom closest to the previous separation surface. The procedure goes on until the number of pivot atoms reaches a specified number. This maximum number of pivot atoms is a parameter of the method, and it is initially chosen in terms of the average density of the system and the width of the layers as obtained from the calculation of the average density profile (i.e. without removing the CWs). If the number of pivot atoms selected is not correct, this will have a direct consequence in the appearance of unphysical shoulders in the intrinsic density profile. In this case the number is changed and the procedure repeated.

The determination of the separation surface in each phase is performed in the following way: first the surface is parameterized by the coefficients of its Fourier expansion, truncated at a maximum wavenumber that corresponds to the ionic radius. These coefficients are then determined by a constrained minimization of the change in the area, where the constraints are that the surface passes through the atomic positions (obviously the number of coefficients in the Fourier expansion must be larger than the number of pivot atoms).

The separation surface thus computed (i.e. characterized by all the Fourier coefficients obtained in the last phase of the construction when the final number of pivots is reached) passes by definition through all of the pivots, and therefore the distance from these atoms to the separation surface is zero, which leads to a $\delta$-function peak in the intrinsic density profile. In the same spirit as the $\sigma_{0}$ value of the preceeding section, it is rather considered that the first peak of the intrinsic density profile should have some width. This is introduced by truncating the Fourier expansion at a smaller cutoff, but keeping the same coefficients determined previously. Each step in the truncation leads to a widening of the first peak, but at some level of truncation the width does not change any more, and we select this level of truncation as the final one.

More details about the procedure can be found in Refs. [39, 40].

\section{Appendix D. Weight function for region selection in the simulated slab}

To study the collective dynamic properties at different depths of the l-In slab, the particles selected in each configuration step need to be only those included inside the region of interest. Given that these type of properties are continuous in time, there cannot be abrupt changes in the particle contribution from one configuration step to the next. For this reason, the contribution of each particle to a given dynamic variable (particle density, particle current, particle number) is weighted by a function whose value is one inside the region of interest and decays smoothly to zero outside this region. The weight function used in this work is,

$$
W(z)=\left\{\begin{array}{lll}
0 & \text { if } & z<z_{\min }-z_{w} \\
0.5+0.5 \cos \left(\frac{z-z_{\min }}{z_{w}} \pi\right) & \text { if } & z_{\min }-z_{w}<z<z_{\min } \\
1 & \text { if } & z_{\min }<z<z_{\max } \\
0.5+0.5 \cos \left(\frac{z-z_{\max }}{z_{w}} \pi\right) & \text { if } & z_{\max }<z<z_{\max }+z_{w} \\
0 & \text { if } & z>z_{\max }+z_{w}
\end{array}\right.
$$

where $z_{\min }$ is the left end of the region, $z_{\max }$ is the right end of the region, and $z_{w}$ is the width of the transition zone where $W(z)$ changes continuously from 1 to 0 or viceversa.

In addition, in order to make a proper comparison with the IXS experimental data, we have also used an exponentially decreasing weight function, $W_{\exp }(z)$, which mimics the exponential decay of the x-ray evanescent wave inside the sample. The rate of the exponential decay is chosen so that it takes a value $1 / e$ at the probing depth desired, which in this specific study is $40 \AA$, the smallest of the probing depths selected in the IXS experimental study of liquid In considered in this work [21, 22]. In fact the decay of this function is not fast enough to neglect the contribution of the other surface of the slab, so in order to eliminate it we have modified the weight function for large enough values of $z$ so that it decays faster and declines to zero before the second surface region is reached.

The form of $W(z)$ for different regions considered in this work, corresponding to the first layer at the interface, the second layer, and the bulk region (a wide section in the center of the slab), and also the form of $W_{\exp }(z)$ can be observed in figure D.1.

\section{Appendix E. Comparison between simulation $S(q, \omega)$ and experimental IXS data}

In order to compare properly the classical $S(q, \omega)$ computed in an MD calculation with the IXS measured intensity, obtained after x-ray photons interact quantum mechanically with the system, it is necessary to perform two corrections. The first one is to obtain a quantum dynamic structure factor, $S_{Q}(q, \omega)$, that fulfills quantum mechanical rules, in particular the detailed balance condition. The 


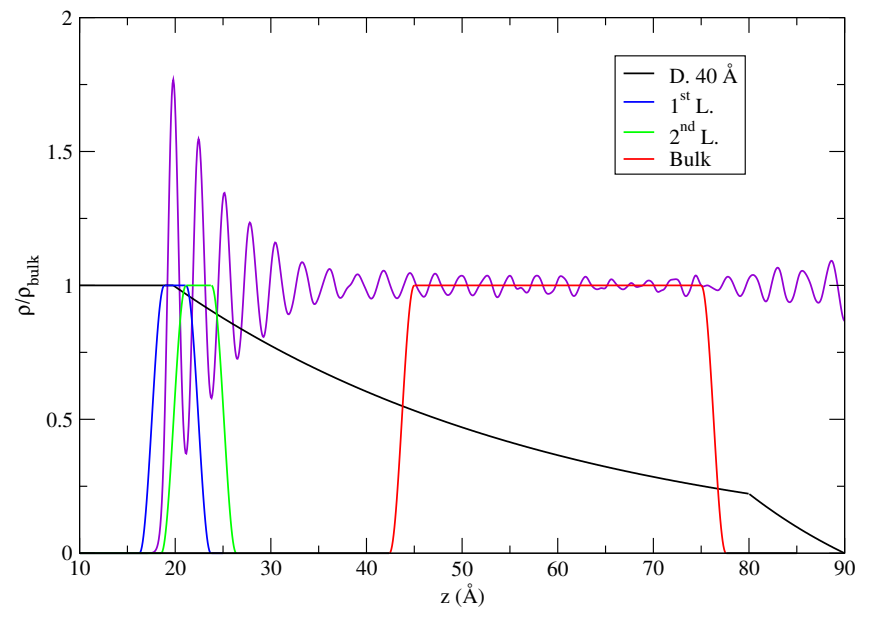

Figure D.1: Regions of the DP (purple) used in the study of the collective dynamics. The windows, calculated by using the weight function in Eq. (D.1), comprise the outmost or first layer (blue), the second layer (green), the bulk region (red), and the exponential decay (black) for a depth of $40 \AA$ to compare with experimental data $[21,22]$.

standard way of performing such conversion is through the formula $[42,43]$

$$
S_{Q}(q, \omega)=\frac{\hbar \beta \omega}{1-e^{-\hbar \beta \omega}} S(q, \omega)
$$

where $\beta=\frac{1}{k_{B} T}$, with $k_{B}$ the Boltzmann constant and $T$ the temperature. The second correction is related to the IXS experimental energy resolution, and it is introduced in the simulation results throught the convolution of $S_{Q}(q, \omega)$ with the experimental resolution function $R(\omega)$, which in IXS experiments takes a Lorentzian form with a width determined by the energy resolution (full width at half maximum), $\Delta E$, of the apparatus. Finally, the measured intensity is usually reported in arbitrary units, so the previously obtained result is multiplied by a normalization factor $A(q)$ selected to bring to a similar magnitude the heights of the inelastic peaks in the IXS measurement and in the calculation:

$$
\begin{gathered}
I(q, \omega)=A(q) \int d \omega^{\prime} S_{Q}\left(q, \omega^{\prime}\right) R\left(\omega-\omega^{\prime}\right), \\
R(\omega)=\frac{c}{\pi\left(\omega^{2}+c^{2}\right)},
\end{gathered}
$$

where $c=\frac{\Delta E}{2}$, and $\Delta E=4.56 \mathrm{ps}^{-1}$ is the energy resolution in the In experiment $[21,22]$.

\section{Appendix F. Fitting of $F(q, t)$ to obtain thermo- physical properties}

In the hydrodynamic region the intermediate scattering function of a bulk liquid is given by the sum of an exponentially decreasing function, related to thermal relax- ation, and a damped oscillating funtion related to sound propagation and attenuation [44]:

$$
\frac{F_{\text {hyd }}(q, t)}{F_{\text {hyd }}(q, 0)}=A_{\mathrm{th}} e^{-\alpha_{\mathrm{th}} t}+A_{s} e^{-\beta_{s} t} \frac{\cos \left(\omega_{s} t-\varphi_{s}\right)}{\cos \varphi_{s}}
$$

with $\alpha_{\text {th }}=D_{T} q^{2}$, where $D_{T}$ is the thermal diffusivity and $\omega_{s}=c_{s} q$, where $c_{s}$ is the adiabatic sound velocity. The expression for $\beta_{s}$ is quadratic in $q$ with a coefficient that involves $D_{T}$ and the longitudinal viscosity $\eta_{L}$, as well as the specific heats ratio $\gamma=C_{P} / C_{V}$. The latter also determines the amplitudes of the two terms, $A_{\mathrm{th}}$ and $A_{s}$. Note that due to these relations, $c_{s}$ can be obtained directly as $\omega_{s} / q$ whereas $\eta_{L}$ can be obtained in terms of $\alpha_{\mathrm{th}}, \beta_{s}, \omega_{s}$, $A_{s}$ and the wavevector $q$.

The extension to larger $q$ values can be performed in several ways. The one we have followed (generalized hydrodynamics) keeps the same structure for $F(q, t)$ as a sum of two functions of the same type as the hydrodynamic ones, but including now a general $q$ dependence in all the thermophysical quantities, i.e. $D_{T}(q), c_{s}(q), \eta_{L}(q)$ and $\gamma(q)$, and consequently on the parameters of the exponential functions, $A_{\mathrm{th}}(q), \alpha_{\mathrm{th}}(q), A_{s}(q), \omega_{s}(q)$ and $\varphi_{s}(q)$, which still have the same relation with the thermophysical $q$-dependent parameters as in the hydrodynamic model.

A second route to the extension to larger $q$ proceeds through the memory function formalism [44] and the model corresponds to expressing the second order memory function of $F(q, t)$ as a sum of several exponential terms. This, in turn, implies that $F(q, t)$ is again a sum of exponentials either real, that correspond to simple exponentially decaying functions, or in complex conjugate pairs, that correspond (one for each pair) to damped oscillatory functions. The total number of exponentials in $F(q, t)$ is two plus the number of exponentials used in the second order memory function. In the analysis of the IXS results for the l-In surface the authors employed this route, by considering two exponentials in the memory function, and correspondingly, four exponentials in $F(q, t)$, two of them real and a complex conjugate pair. Therefore their oscillating term is just like the generalized hydrodynamic one, while the non-oscillating term had two exponentials instead of one in our model.

When coming to the slab configuration, an additional term has to be added to take into account the contribution of capillary waves. In our model we used another damped oscillatory function corresponding to a propagating wave. In the IXS analysis, due to the width of the resolution function, it was not possible to resolve the peaks associated to $\mathrm{CW}$ propagation, and therefore a simple exponentially decaying function was used to model this contribution.

All in all, our model for the $F(q, t)$ in the slab contains one exponentially decaying term and two damped oscillatory functions, one for sound and one for CWs. Instead, the IXS measurements were analyzed in terms of an expression for $F(q, t)$ containing one damped oscilla- 
tory function for sound and three exponentially decaying terms, one of them associated to CWs.

\section{References}

[1] C. P. Sebastian, C. D. Malliakas, M. Chondroudi, I. Schellenberg, S. Rayaprol, R.-D. Hoffmann, R. Pttgen, M. G. Kanatzidis, Indium Flux-Growth of $\mathrm{Eu}_{2} \mathrm{AuGe}_{3}$ : A New Germanide with an $\mathrm{AlB}_{2}$ Superstructure, Inorg. Chem. 49 (2010) 9574-9580.

[2] Y. D. Kim, J. Hone, Screen printing of 2D semiconductors, Nature 544 (2017) 167-168.

[3] K. Wang, K. Jiang, B. Chung, T. Ouchi, P. J. Burke, D. A. Boysen, D. J. Bradwell, H. Kim, U. Muecke, D. R. Sadoway, Lithium-antimony-lead liquid metal battery for grid-level energy storage, Nature 514 (2014) 348-350.

[4] H. Kim, D. A. Boysen, J. M. Newhouse, B. L. Spatocco, B. Chung, P. J. Burke, D. J. Bradwell, K. Jiang, A. A. Tomaszowska, K. Wang, W. Wei, L. A. Ortiz, S. A. Barriga, S. M. Poizeau, D. R. Sadoway, Liquid Metal Batteries: Past, Present, and Future, Chem. Rev. 113 (2013) 2075-2099.

[5] H. Li, H. Yin, K. Wang, S. Cheng, K. Jiang, D. R. Sadoway, Liquid Metal Electrodes for Energy Storage Batteries, Adv. Energy Mater. 6 (2016) 1600483.

[6] K. Khoshmanesh, S.-Y. Tang, J. Y. Zhu, S. Schaefer, A. Mitchell, K. Kalantar-Zadeh, M. D. Dickey, Liquid metal enabled microfluidics, Lab Chip 17 (2017) 974-993.

[7] J. Y. Zhu, S.-Y. Tang, K. Khoshmanesh, K. Ghorbani, An Integrated Liquid Cooling System Based on Galinstan Liquid Metal Droplets, ACS Appl. Mater. Interfaces 8 (2016) 2173-2180.

[8] G. Li, D.-W. Lee, An advanced selective liquid-metal plating technique for stretchable biosensor applications, Lab Chip 17 (2017) 3415-3421.

[9] T. Lu, E. J. Markvicka, Y. Jin, C. Majidi, Soft-Matter Printed Circuit Board with UV Laser Micropatterning, ACS Appl. Mater. Interfaces 9 (2017) 22055-22062.

[10] Y. Lu, Q. Hu, Y. Lin, D. B. Pacardo, C. Wang, W. Sun, F. S. Ligler, M. D. Dickey, Z. Gu, Transformable liquid-metal nanomedicine, Nat. Commun. 6 (2015) 10066.

[11] T. Daeneke, K. Khoshmanesh, N. Mahmood, I.A. de Castro, D. Esrafilzadeh, S. J. Barrow, M. D. Dickey, K. Kalanta-zadeh, Liquid metals: fundamentals and applications in chemistry, Chem. Soc. Rev, 47 (2018) 4073.

[12] M. P. D'Evelyn, S. A. Rice, Structure in the Density Profile at the Liquid-Metal-Vapor Interface, Phys. Rev. Lett. 47 (1981) 1844.

[13] O.M. Magnussen, B.M. Ocko, M. J. Regan, K. Penanen, P.S. Pershan, M. Deutsch, X-Ray Reflectivity Measurements of Surface Layering in Liquid Mercury, Phys. Rev. Lett. 74 (1995) 4444.

[14] M. J. Regan, E. H. Kawamoto, S. Lee, P. S. Pershan, N. Maskil, M. Deutsch, O. M. Magnussen, B. M. Ocko, L. E. Berman, Surface Layering in Liquid Gallium: An X-Ray Reflectivity Study, Phys. Rev. Lett. 75 (1995) 2498.

[15] H. Tostmann, E. DiMasi, P. S. Pershan, B. M. Ocko, O. G. Shpyrko, M. Deutsch, Surface structure of liquid metals and the effect of capillary waves: X-ray studies on liquid indium, Phys. Rev. B 59 (1999) 783.

[16] O. G. Shpyrko, P. Huber, A. Y.Grigoriev, P. S. Pershan, B. Ocko, H. Tostmann, M. Deutsch, X-ray study of the liquid potassium surface:Structure and capillary wave excitations, Phys. Rev. B 67 (2003) 115405.

[17] O. G. Shpyrko, A. Y.Grigoriev, C. Steimer, P. S.Pershan, B. Lin, M. Meron, T. Graber, J. Gerbhardt, B. Ocko, M. Deutsch, Anomalous layering at the liquid Sn surface, Phys. Rev. B 70 (2004) 224206.

[18] P. S. Pershan, S. E. Stoltz, O. G. Shpyrko, M. Deutsch, V. S. K. Balagurusamy, M. Meron, B. Lin, R. Streitel, Surface structure of liquid Bi and Sn: An x-ray reflectivity study, Phys. Rev. B 79 (2009) 115417.
[19] P. S. Pershan, M. L. Schlossman, Liquid Surfaces and Interfaces. Synchrotron X-ray Methods, Cambridge, New York, 2012.

[20] T. Scopigno, G. Ruocco, F. Sette, Microscopic dynamics in liquid metals: The experimental point of view, Rev. Mod. Phys. 77 (2005) 881.

[21] H. Reichert, F. Bencivenga, B. Wehinger, M. Krisch, F. Sette, H. Dosch, High-Frequency Subsurface and Bulk Dynamics of Liquid Indium, Phys. Rev. Lett. 98 (2007) 096104.

[22] B. Wehinger, M. Krisch, H. Reichert, High-frequency dynamics in the near-surface region studied by inelastic x-ray scattering: the case of liquid indium, New J. Phys. 13 (2011) 023021.

[23] S. Iarlori, P. Carnevali, F. Ercolessi, E. Tosatti, Dynamics of a Liquid-Metal Surface, Europhys. Lett. 10 (1989) 329.

[24] P. Hohenberg, W. Kohn, Inhomogeneous Electron Gas, Phys. Rev. 136 (1964) B864.

[25] W. Kohn, L. J. Sham, Self-Consistent Equations Including Exchange and Correlation Effects, Phys. Rev. 140 (1965) A1133.

[26] D. J. Gonzalez, L. E. Gonzalez, J. M. Lopez, M. J. Stott, Dynamical properties of liquid Al near melting: An orbital-free molecular dynamics study, Phys. Rev. B 65 (2002) 184201.

[27] D. J. Gonzalez, L. E. Gonzalez, J. M. Lopez, M. J. Stott, Orbital free ab initio molecular dynamics study of liquid Al near melting, J. Chem. Phys. 115 (2001) 2373.

[28] E. Chacon, J. E. Alvarellos, P. Tarazona, Nonlocal kinetic energy functional for nonhomogeneous electron systems, Phys. Rev. B 32 (1985) 7868.

[29] P. Garcia-Gonzalez, J. E. Alvarellos, E. Chacon, Kinetic-energy density functional: Atoms and shell structure, Phys. Rev. A 54 (1996) 1897.

[30] P. Garcia-Gonzalez, J. E. Alvarellos, E. Chacon, Nonlocal kinetic-energy-density functionals, Phys. Rev. B 53 (1996) 9509.

[31] P. Garcia-Gonzalez, J. E. Alvarellos, E. Chacon, Nonlocal symmetrized kinetic-energy density functional: Application to simple surfaces, Phys. Rev. B 57 (1998) 4857.

[32] D. M. Ceperley, B. J. Alder, Ground State of the Electron Gas by a Stochastic Method, Phys. Rev. Lett. 45, (1980) 566.

[33] L. Dagens, Application de la méthode de l'atome neutre auxiliaire au calcul de l'énergie des métaux simples, J. Phys. (Paris) 34 (1973) 879.

[34] L. E. Gonzalez, A. Meyer, M. P. Iniguez, D. J. Gonzalez, Liquid structure of the alkaline-earth metals, Phys. Rev. E 47 (1993) 4120 .

[35] B. G. del Rio, L. E. Gonzlez, Orbital free ab initio simulations of liquid alkaline earth metals: from pseudopotential construction to structural and dynamic properties, J. Phys.: Condens. Matter 26 (2014) 465102.

[36] S. Kirkpatrick, C. D. Gelatt Jr., M. P. Vecchi, Optimization by Simulated Annealing, Science 13 (1983) 4598.

[37] R. E. Eckert, H. G. Drickamer, Diffusion in Indium Near the Melting Point, J. Chem. Phys. 20, (1952) 13-17.

[38] Y. Waseda, The structure of Non-crystalline Materials, McGraw-Hill, New York, 1980.

[39] E. Chacon, P. Tarazona, Intrinsic Profiles beyond the Capillary Wave Theory: A Monte Carlo Study, Phys. Rev. Lett. 91 (2003) 166103.

[40] E. Chacón, P. Tarazona, L. E. González, Intrinsic structure of the free liquid surface of an alkali metal, Phys. Rev. B 74 (2006) 224201.

[41] P. Gianozzi et al, QUANTUM ESPRESSO: a modular and open-source software project for quantum simulations of materials, J. Phys.: Condens. Matter 21 (2009) 395502.

[42] T. Scopigno, U. Balucani, G. Ruocco, F. Sette, Collective dynamics of liquid aluminum probed by inelastic $x$-ray scattering, Phys. Rev. E 63, (2000) 011210.

[43] T. Scopigno, U. Balucani, G. Ruocco, F. Sette, Evidence of Two Viscous Relaxation Processes in the Collective Dynamics of Liquid Lithium, Phys. Rev. Lett. 85 (2000) 4076.

[44] U. Bafile, E. Guarini, F. Barocchi, Collective acoustic modes as renormalized damped oscillators: Unified description of neutron and $x$-ray scattering data from classical fluids, Phys. Rev. E 73 (2006) 061203. 